\title{
Special issue on extracellular space
}

\author{
Reinoud Maex \\ Ecole Normale Supérieure, rue d'Ulm 29, Paris 75005, France \\ E-mail: reinoud.maex@ens.fr
}

The vast majority of the brain's electrical signals are recorded from, or filtered by, extracellular space, yet our knowledge of this compartment is rather limited as compared to that of intracellular space and the neuronal membrane.

How should extracellular space be modelled? How does the way we model it change our interpretation of the recorded signals, or even the dynamics of the neuron models? Finally, is extracellular space (including the glial cells) not more than the neurons' Cinderella, or can it claim its own role in excitability and cognition? These are the questions that have occupied the contributors to this special issue on Extracellular Space.

The simplest model is that of extracellular space as a mere electrolytic solution, a potentially homogeneous and purely resistive volume conductor [7]. Bédard, Gomes, Bal and Destexhe [1] examine how capacitive and diffusive properties of extracellular space - the ions carrying the current being trapped by membranes and macromolecules, or escaping via the electrode track - may alter the power spectrum of the recorded signals, and more particularly how these effects may induce the $1 / f$ scaling that they and others typically observe in extracellular and surface recordings.

Pods [8] compares changes in the shape of the action potential recorded in extracellular space when the simulated physical model is not simply that of a volume conductor, but takes also account of electrodiffusion (the Nernst-Planck equation) and free charges (adding the Poisson equation and giving up electro-neutrality within the Debye layer).

In a combined analytical-numerical tour-de-force, Xiang, Liu, Tang and Yan [10] formulate the full equations of motion of the ions carrying the local-circuit membrane currents, including not only ion transport along both the intra- and extracellular compartments but also across the ion channels. Their model is an extension of the electro-diffusive cable model [9] to extracellular space, and predicts the occurrence of teraHz oscillations owing to Coulomb interactions of the mobile ions with fixed charges in the wall of the ion channel.

How do ions and ligands find their way through extracellular space and reach their channels, and receptors, on the membrane? The classical view is that diffusion does the job, and that thermal agitation enhances the probability of a ligand hitting its receptor to such a degree that increasing the density of receptors would hardly be profitable [2]. Two papers in this issue [3,6] review older and recent work indicating that the extracellular electrolytic solution (or gel or colloid) is much more organized than commonly conceived. Pereira [6] suggest that extracellular space may have a guiding role, for de Lima and Hanke [3] it is excitable and active itself. 
There are further some common threads through this issue, such as the Nernst-Planck equation [5, 8,10], vibrations and resonances [6,10], electrode polarization $[1,3,5], 1 / f$ power scaling [1,5], etc. Finally, Harris Bozer, Uhelski and Li [4] review methodological issues of local field potential recordings, and their applications.

\section{References}

[1] C. Bédard, J.-M. Gomes, T. Bal and A. Destexhe, A framework to reconcile frequency scaling measurements, from intracellular recordings, local-field potentials, up to EEG and MEG signals, Journal of Integrative Neuroscience 16 (2017), $3-18$.

[2] H.C. Berg and E.M. Purcell, Physics of chemoreception, Biophysical Journal 20 (1977), 193-219. doi:10.1016/S00063495(77)85544-6.

[3] V.M.F. de Lima and W. Hanke, Extracellular matrix and its role in conveying glial/neural interactions in health and disease, Journal of Integrative Neuroscience 16 (2017), 93-106.

[4] A.L. Harris Bozer, M.L. Uhelski and A.-L. Li, Extrapolating meaning from local field potential recordings, Journal of Integrative Neuroscience 16 (2017), 107-126.

[5] R. Maex, On the Nernst-Planck equation, Journal of Integrative Neuroscience 16 (2017), 73-91.

[6] A. Pereira, Jr, Astroglial hydro-ionic waves guided by the extracellular matrix: An exploratory model, Journal of Integrative Neuroscience 16 (2017), 57-72.

[7] R. Plonsey, Volume conductor fields of action currents, Biophysical Journal 4 (1964), 317-328. doi:10.1016/S00063495(64)86785-0.

[8] J. Pods, A comparison of computational models for the extracellular potential of neurons, Journal of Integrative Neuroscience 16 (2017), 19-32.

[9] N. Qian and T.J. Sejnowski, An electro-diffusion model for computing membrane potentials and ionic concentrations in branching dendrites, spines and axons, Biological Cybernetics 62 (1989), 1-15. doi:10.1007/BF00217656.

[10] Z.X. Xiang, G.Z. Liu, C.X. Tang and L.X. Yan, A model of ion transport processes along and across the neuronal membrane, Journal of Integrative Neuroscience 16 (2017), 33-55. 